\title{
The reaction of carbon disulphide with $\alpha$-haloketones and primary amines in the presence of potassium iodide as catalyst
}

\author{
JAVAD SAFAEI-GHOMI ${ }^{\mathrm{a}, *}$, FARIBA SALIMI ${ }^{\mathrm{a}}$ and ALI RAMAZANI ${ }^{\mathrm{b}}$ \\ ${ }^{a}$ Department of Chemistry, Faculty of Sciences, University of Kashan, Kashan, 51167, IR, Iran \\ ${ }^{\mathrm{b}}$ Chemistry Department, Zanjan University, P O Box 45195-313, Zanjan, Iran \\ e-mail: safaei@kashanu.ac.ir
}

MS received 3 June 2012; revised 23 March 2013; accepted 4 July 2013

\begin{abstract}
A simple, mild and convenient method has been developed for the synthesis of 3,4,5-trialkyl-1,3thiazole-2 $(3 H)$-thione derivatives through one pot three-component reaction between a primary amine, carbon disulphide, and $\alpha$-haloketone in the presence of potassium iodide at room temperature conditions. The products were obtained with excellent yield and appropriate reaction times. This reaction represents a rapid and unprecedented route to the described molecules that have biological specifications.
\end{abstract}

Keywords. carbon disulphide; $\alpha$-haloketone; primary amines; potassium iodide; thiazole; nucleophilic addition.

\section{Introduction}

Multicomponent reactions (MCR) have appeared as an efficient and powerful tool in modern synthetic organic chemistry due to their valued features such as atom economy, straightforward reaction design, and the opportunity to construct target compounds by the introduction of several diversity elements in a single chemical event. Since all the organic reagents employed are consumed and incorporated into the target compound, purification of products resulting from MCR is also simple. ${ }^{1} \mathrm{MCR}$, leading to interesting heterocyclic scaffolds, are especially useful for the construction of diverse chemical libraries of 'druglike' molecules. The isocyanide-based MCRs are very important in this area. ${ }^{2-4}$ Among the known multicomponent reactions to date, the most valuable reactions are those based on isocyanides. Isocyanide-based multicomponent reactions (abbreviated to IMCRs by Ugi and Dömling) by virtue of their synthetic potential, their inherent atom efficiency, convergent nature, ease of implementation, and the generation of molecular diversity, have attracted considerable attention because of the advantages that they offer to the field of combinatorial chemistry. ${ }^{5-9}$

Organic compounds containing five-membered aromatic heterocyclic rings constitute a wide range of compounds in the nature and often play an important role in various biochemical processes. As a result, aromatic heterocycles such as thiophenes, benzothiophene

*For correspondence derivatives, and their reduced forms are significant structural fragments in many pharmaceutical and chemical compounds. Thiazoline and thiazole compounds have been found to represent nematocidal, insecticidal, antibacterial, antifungal, antiviral, and antioxidant activity. ${ }^{10-15}$ Tetrahydrothiophene is an important building block of a large quantity of compounds that are very interesting from the viewpoint of biological activity. Its derivatives have showed antisecretory and antiulcer activities. ${ }^{16}$ Because of the potential use of thiophenes and annulated thiophenes as pharmaceuticals, conjugated polymers, organic conductors, semiconductors and light emitting devices, their synthesis are of special interest. ${ }^{17-22}$ Alkylation of an intermediate dimetaloketene dithioacetal is a general strategy employed for the synthesis of alkylthiothiophenes or thienothiophenes. ${ }^{23-26}$ As part of our ongoing program to develop efficient and robust methods for the preparation of heterocyclic compounds, ${ }^{27-39}$ we have prepared 3-alkyl4-phenyl-1,3-thiazole-2(3H)-thionewithout catalyst in our last report ${ }^{40}$ and now we wish to report the preparation of a 3, 4, 5- trialkyl-1, 3- thiazole- $2(3 H)$ - thione derivatives through a one-pot multicomponent condensation reaction (MCR) in the presence of potassium iodide (scheme 1).

\section{Experimental}

Starting materials and solvents were obtained from Merck (Germany) and Fluka (Switzerland) and were 


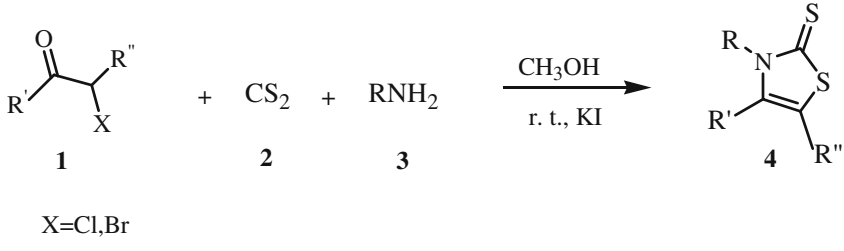

Scheme 1. Three-component synthesis of 3,4,5-trialkyl1,3-thiazole-2(3H)-thione derivatives 4 in the presence of potassium iodide.

used without further purification. TLC and NMR spectroscopy were used to follow the reactions. Melting points were measured on an Electrothermal 9100 apparatus and are uncorrected. IR spectra were measured on a Jasco 6300 FTIR spectrometer. ${ }^{1} \mathrm{H}$ and ${ }^{13} \mathrm{C}$ NMR spectra were measured $\left(\mathrm{CDCl}_{3}\right.$ solution) with a BRUKER DRX-250 Avance spectrometer at 250.0 and $62.5 \mathrm{MHz}$, respectively. Elemental analyses were performed using a Heraeus CHN-O-Rapid analyzer. Preparative layer chromatography (PLC) plates were prepared from Merck silica gel $\left(\mathrm{F}_{254}\right)$ powder.

\subsection{General procedure for the preparation of compounds 4}

The mixture of $\alpha$-haloketone $1(1 \mathrm{mmol})$ and potassium iodide $0.1 \mathrm{~g}(0.6 \mathrm{mmol})$ in $\mathrm{CH}_{3} \mathrm{OH}(7 \mathrm{ml})$ was stirred for $30 \mathrm{~min}$ and then the solution of primary amine $(1 \mathrm{mmol})$ and carbon disulphide $(1 \mathrm{mmol})$ was added, and the mixture was stirred for $24 \mathrm{~h}$. The solvent was removed under reduced pressure and the viscous residue was purified by preparative layer chromatography (PLC) [silica gel $\left(\mathrm{F}_{254}\right)$ powder; petroleum etherethyl acetate (10:2)]. The solvent was removed under reduced pressure and the products were obtained. The characterization data of the compounds are given below.

2.1a 3-(2-Furylmethyl)-4,5- diphenyl-1,3- thiazole2(3H)- thione (4a): White powder, (yield: $85 \%$ ). IR (neat): $v=3150,3000,1625,1600,1475,1300$, $1100 \mathrm{~cm}^{-1} .{ }^{1} \mathrm{H}$ NMR $\left(250 \mathrm{MHz}, \mathrm{CDCl}_{3}\right) \delta(\mathrm{ppm}): 5.34$ $\left(\mathrm{s}, 2 \mathrm{H}, \mathrm{CH}_{2}\right), 6.12(\mathrm{~s}, 1 \mathrm{H}, \mathrm{CH}$ of furan), $6.24(\mathrm{~s}$, $1 \mathrm{H}, \mathrm{CH}$ of furan), $7.00-7.47\left(\mathrm{~m}, 11 \mathrm{H}, \mathrm{CH}_{\text {arom }}\right.$ and $\mathrm{CH}$ of furan). ${ }^{13} \mathrm{CNMR}\left(62.5 \mathrm{MHz}, \mathrm{CDCl}_{3}\right) \delta$ (ppm): $44.66\left(\mathrm{CH}_{2}\right), 109.59,110.47$ ( $\mathrm{CH}$ of furan), 125.00 (C of alkene), 128.02, 128.23, 128.64, 129.10, 130.00, 130.87 (10CH), 130.05, 130.19 (C), 138.63 (C of alkene), 142.04 (CH of furan), 147.94 (C of furan), $186.80(\mathrm{C}=\mathrm{S}) . \mathrm{MS}, m / z(\%): 349$ (80), 316 (48), 210 (52), 171 (80), 151 (86), 105 (84), 81 (100), 69 (40), 53 (48). Analysis of $\mathrm{C}_{20} \mathrm{H}_{15} \mathrm{NOS}_{2}$ (349.47). (\% calculation/found): C: 68.74/68.80, H: 4.33/4.39, $\mathrm{N}: 4.01 / 3.95$. 2.1b 3-Benzyl-4- (4- methoxyphenyl)- 1,3-thiazole2(3H)- thione $(\mathbf{4 b})$ : White powder, (yield: $87 \%$ ). IR (neat): $v=3150,3000,1625,1600,1475,1300$, $1100 \mathrm{~cm}^{-1} \cdot{ }^{1} \mathrm{H}$ NMR $\left(250 \mathrm{MHz}, \mathrm{CDCl}_{3}\right) \delta(\mathrm{ppm}): 3.82$ $\left(\mathrm{s}, 3 \mathrm{H}, \mathrm{OCH}_{3}\right), 5.39\left(\mathrm{~s}, 2 \mathrm{H}, \mathrm{CH}_{2}\right), 6.45(\mathrm{~s}, 1 \mathrm{H}$, $\mathrm{CH}$ of alkene), 6.82-7.21 (m, 9H, $\left.\mathrm{CH}_{\text {arom }}\right) .{ }^{13} \mathrm{CNMR}$ $\left(62.5 \mathrm{MHz}, \mathrm{CDCl}_{3}\right) \delta(\mathrm{ppm}): 50.82\left(\mathrm{CH}_{2}\right), 55.40$ $\left(\mathrm{OCH}_{3}\right), 108.65$ (CH of alkene), 114.01, 126.98, 127.52, 128.49, 130.89 (9CH), 122. 79, 135.42, 160.67 (3C), 144.75 ( $\mathrm{C}$ of alkene), $188.86(\mathrm{C}=\mathrm{S}) . \mathrm{MS}$, m/z (\%): 313 (56), 297 (80), 280 (36), 229 (44), 206 (72), 149 (44), 135 (64), 91 (92), 69 (100), 57 (56). Analysis of $\mathrm{C}_{17} \mathrm{H}_{15} \mathrm{NOS}_{2}$ (313.44). (\% calculation/ found): C: $65.14 / 65.08, \mathrm{H}: 4.82 / 4.88, \mathrm{~N}: 4.47 / 4.41$.

2.1c 4-(4-Methoxyphenyl)-3-(4- methylbenzyl)- 1,3thiazole-2(3H)- thione $(\mathbf{4 c )}$ : White powder, (yield: $90 \%$ ). IR (neat): $v=3100,3000,1650,1600,1475$, $1200 \mathrm{~cm}^{-1} .{ }^{1} \mathrm{H}$ NMR $\left(250 \mathrm{MHz}, \mathrm{CDCl}_{3}\right) \delta(\mathrm{ppm}): 2.29$ (s, $\left.3 \mathrm{H}, \mathrm{CH}_{3}\right), 3.82$ (s, 3H, $\left.\mathrm{OCH}_{3}\right), 4.82\left(\mathrm{~s}, 2 \mathrm{H}, \mathrm{CH}_{2}\right)$, $5.94(\mathrm{~s}, 1 \mathrm{H}, \mathrm{CH}$ of alkene), 6.84-7.26 $(\mathrm{m}, 8 \mathrm{H}, \mathrm{CH}$ arom). ${ }^{13} \mathrm{CNMR}\left(62.5 \mathrm{MHz}, \mathrm{CDCl}_{3}\right) \delta$ (ppm): 21.10 $\left(\mathrm{CH}_{3}\right), 46.95\left(\mathrm{CH}_{2}\right), 55.36\left(\mathrm{OCH}_{3}\right), 98.28(\mathrm{CH}$ of alkene), 113.94, 127.07, 129.19, $130.53(8 \mathrm{CH}), 123.77$, 133.59, 137.09, 160.29 (4C), 137.61 (C of alkene), $172.85(\mathrm{C}=\mathrm{S})$. Analysis of $\mathrm{C}_{18} \mathrm{H}_{17} \mathrm{NOS}_{2}$ (327.46). (\% calculation/found): C: 66.02/66.06, H: 5.23/5.17, $\mathrm{N}: 4.28 / 4.22$.

2.1d 3-(2-Furylmethyl)-4- (4- methoxyphenyl)- 1, 3thiazole-2(3H)- thione $(\mathbf{4 d})$ : White powder, (yield: $94 \%)$. IR (neat): $v=3150,3000,1625,1600,1475$, $1300,1100 \mathrm{~cm}^{-1} .{ }^{1} \mathrm{H} \mathrm{NMR}\left(250 \mathrm{MHz}, \mathrm{CDCl}_{3}\right) \delta(\mathrm{ppm})$ : $3.85\left(\mathrm{~s}, 3 \mathrm{H}, \mathrm{OCH}_{3}\right), 4.80\left(\mathrm{~s}, 2 \mathrm{H}, \mathrm{CH}_{2}\right), 5.95(\mathrm{~s}, 1 \mathrm{H}, \mathrm{CH}$ of alkene), 6.03, 6.24 (s, 2CH, $\mathrm{CH}$ of furan), 6.92-7.29 (m, $5 \mathrm{H}, \mathrm{CH}_{\text {arom }}$ and $\mathrm{CH}$ of furan). ${ }^{13} \mathrm{CNMR}(62.5 \mathrm{MHz}$, $\left.\mathrm{CDCl}_{3}\right) \delta(\mathrm{ppm}): 40.48\left(\mathrm{CH}_{2}\right), 55.38\left(\mathrm{OCH}_{3}\right), 98.32$, (CH of alkene), 108.60, 110.44, 142.21 (3CH of furan), 114.04, 130.61 (4CH), 123.50, 160.37 (2C), 137.22 (C of alkene), 149.36 ( $\mathrm{C}$ of furan), $172.55(\mathrm{C}=\mathrm{S})$. Analysis of $\mathrm{C}_{15} \mathrm{H}_{13} \mathrm{NO}_{2} \mathrm{~S}_{2}$ (303.40). (\% calculation/found): C: 59.38/59.32, H: 4.32/4.26, N: 4.62/4.56.

2.1e 3-(2-Methoxybenzyl)-4-(4- methoxyphenyl)- 1, 3-thiazole-2(3H)-thione (4e): White powder, (yield: $87 \%$ ). IR (neat): $v=3100,3000,1600,1475$, $1200 \mathrm{~cm}^{-1} .{ }^{1} \mathrm{H}$ NMR $\left(250 \mathrm{MHz}, \mathrm{CDCl}_{3}\right) \delta(\mathrm{ppm})$ : 3.67, $3.79\left(2 \mathrm{~s}, 6 \mathrm{H}, 2 \mathrm{OCH}_{3}\right), 4.87\left(\mathrm{~s}, 2 \mathrm{H}, \mathrm{CH}_{2}\right), 5.99$ (s, 1H, CH of alkene), 6.74-7.22 (m, 8H, CH arom). ${ }^{13} \mathrm{CNMR}\left(62.5 \mathrm{MHz}, \mathrm{CDCl}_{3}\right) \delta$ (ppm): $42.72\left(\mathrm{CH}_{2}\right)$, 55.12, $55.31\left(2 \mathrm{OCH}_{3}\right), 98.07$ ( $\mathrm{CH}$ of alkene), 109.94, $113.85,120.55,126.78,128.30,130.08(8 \mathrm{CH}), 123.81$, 
124.64, 156.31, 160.14 (4C), 137.99 (C of alkene), $172.79(\mathrm{C}=\mathrm{S})$. Analysis of $\mathrm{C}_{18} \mathrm{H}_{17} \mathrm{NO}_{2} \mathrm{~S}_{2}$ (343.46). (\% calculation/found): C: 62.94/62.88, H: 4.99/4.93, $\mathrm{N}: 4.08 / 4.02$.

2.1f 3- (2- Methoxybenzyl)- 4-methyl-1, 3-thiazole2(3H)-thione $(\mathbf{4 f})$ : White powder, (yield: $88 \%$ ). IR (neat): $v=3100,3000,1600,1475,1200 \mathrm{~cm}^{-1} \cdot{ }^{1} \mathrm{H}$ NMR $\left(250 \mathrm{MHz}, \mathrm{CDCl}_{3}\right) \delta(\mathrm{ppm}): 2.01\left(\mathrm{~s}, 3 \mathrm{H}, \mathrm{CH}_{3}\right)$, $3.86\left(\mathrm{~s}, 3 \mathrm{H}, \mathrm{OCH}_{3}\right), 4.95\left(\mathrm{~s}, 2 \mathrm{H}, \mathrm{CH}_{2}\right), 5.78(\mathrm{~s}, 1 \mathrm{H}$, $\mathrm{CH}$ of alkene), 6.85-6.90 (m, 4H, $\left.\mathrm{CH}_{\text {arom }}\right) .{ }^{13} \mathrm{CNMR}$ $\left(62.5 \mathrm{MHz}, \mathrm{CDCl}_{3}\right) \delta(\mathrm{ppm}): 15.12\left(\mathrm{CH}_{3}\right), 41.21\left(\mathrm{CH}_{2}\right)$, $55.24\left(\mathrm{OCH}_{3}\right), 97.48(\mathrm{CH}$ of alkene $), 110.11,120.79$, $126.61,128.55$ (4CH), 126.50, 158.61 (2C), 134.46 (C of alkene), $185.52(\mathrm{C}=\mathrm{S})$. Analysis of $\mathrm{C}_{12} \mathrm{H}_{13} \mathrm{NOS}_{2}$ (251.37). (\% calculation/found): C: $57.34 / 57.40, \mathrm{H}$ : $5.21 / 5.15$, N: 5.57/5.51.

2.1g 3-(2-Methoxybenzyl)-4, 5-dimethyl-1, 3- thiazole2(3H)-thione $\mathbf{( 4 g )}$ : White powder, (yield: $90 \%$ ). IR (neat): $v=3100,3000,1600,1475,1200 \mathrm{~cm}^{-1} \cdot{ }^{1} \mathrm{H}$ NMR $\left(250 \mathrm{MHz}, \mathrm{CDCl}_{3}\right) \delta(\mathrm{ppm}): 1.90,2.09$ (2s, $\left.6 \mathrm{H}, 2 \mathrm{CH}_{3}\right), 3.85\left(\mathrm{~s}, 3 \mathrm{H}, \mathrm{OCH}_{3}\right), 4.91\left(\mathrm{~s}, 2 \mathrm{H}, \mathrm{CH}_{2}\right)$,
6.79-7.21 (m, 4H, $\left.\mathrm{CH}_{\text {arom }}\right) .{ }^{13} \mathrm{CNMR}(62.5 \mathrm{MHz}$, $\left.\mathrm{CDCl}_{3}\right) \delta(\mathrm{ppm}): 11.22\left(\mathrm{CH}_{3}\right), 12.12\left(\mathrm{CH}_{3}\right), 41.44$ $\left(\mathrm{CH}_{2}\right), 55.29\left(\mathrm{OCH}_{3}\right), 108.28$ (C of alkene), 110.06, $120.78,126.61,128.40(4 \mathrm{CH}), 124.50,158.61$ (C), 142.46 ( $\mathrm{C}$ of alkene), $171.92(\mathrm{C}=\mathrm{S})$. Analysis of $\mathrm{C}_{13} \mathrm{H}_{15} \mathrm{NOS}_{2}$ (265.39). (\% calculation/found): C: 58.83/58.77, H: 5.70/5.76, N: 5.28/5.22.

2.1h 3-(2-Furylmethyl)-4, 5-dimethyl-1, 3-thiazole2(3H)-thione (4h): White powder, (yield: $92 \%$ ). IR (neat): $v=3100,3000,1600,1475,1200 \mathrm{~cm}^{-1}$. ${ }^{1} \mathrm{H}$ NMR $\left(250 \mathrm{MHz}, \mathrm{CDCl}_{3}\right) \quad \delta(\mathrm{ppm}): 2.12,2.28$ $\left(2 \mathrm{~s}, 6 \mathrm{H}, 2 \mathrm{CH}_{3}\right), 5.40\left(\mathrm{~s}, 2 \mathrm{H}, \mathrm{CH}_{2}\right), 6.32,6.47,7.33$ (3CH of furan). ${ }^{13} \mathrm{CNMR}\left(62.5 \mathrm{MHz}, \mathrm{CDCl}_{3}\right) \delta$ (ppm): $11.63\left(\mathrm{CH}_{3}\right), 12.78\left(\mathrm{CH}_{3}\right), 43.75\left(\mathrm{CH}_{2}\right), 110.05$, $110.74,142.30$ (3CH of furan), 117.32, 134.52 (2C of alkene), 148.17 ( $\mathrm{C}$ of furan), $186.92(\mathrm{C}=\mathrm{S})$. Analysis of $\mathrm{C}_{10} \mathrm{H}_{11} \mathrm{NOS}_{2}$ (225.33). (\% calculation/found): $\mathrm{C}$ : 53.30/53.36, H: 4.92/4.98, N: 6.22/6.28.

2.11 4,5 -Dimethyl-3-(4- methylbenzyl)-1,3- thiazole2(3H)-thione (4i): White powder, (yield: $90 \%$ ). IR (neat): $v=3100,3000,1600,1475,1200 \mathrm{~cm}^{-1}$.<smiles>[R]C(=O)C([Y])[I-][Na]</smiles><smiles>C=C[AsH]C(=S)NC(C)(C)C</smiles><smiles>[R]NC(=[SH+])SCCCCC([R7])C([R])=O</smiles><smiles>[R]NC(=S)SC([R])C([R])[O-]</smiles>

(1)



Scheme 2. Proposed mechanism for the formation of 3,4,5-trialkyl-1,3thiazole-2(3H)-thione derivatives $\mathbf{4}$ in the presence of potassium iodide. 
Table 1. Synthesis of 3,4,5-trialkyl-1,3-thiazole-2(3H)-thione derivatives 4a-i in the presence of potassium iodide.

\begin{tabular}{|c|c|c|c|c|c|}
\hline Entry & $\mathrm{R}$ & $\mathrm{R}^{\prime}$ & $\mathrm{R}^{\prime \prime}$ & Product & Yield (\%) \\
\hline $4 a$ & 2- Furylmethyl & Phenyl & Phenyl & & 92 \\
\hline $4 b$ & Benzyl & 4- Methoxy phenyl & $\mathrm{H}$ & & 93 \\
\hline $4 c$ & 4- Methyl benzyl & 4- Methoxy phenyl & $\mathrm{H}$ & & 94 \\
\hline $4 d$ & 2- Furylmethyl & 4- Methoxy phenyl & $\mathrm{H}$ & & 95 \\
\hline $4 e$ & 2- Methoxy benzyl & 4- Methoxy phenyl & $\mathrm{H}$ & & 90 \\
\hline $4 f$ & 2- Methoxy benzyl & Methyl & $\mathrm{H}$ & & 90 \\
\hline $4 g$ & 2- Methoxy benzyl & Methyl & Methyl & & 92 \\
\hline
\end{tabular}


Table 1. (continued).

4h 2- Furylmethyl $\quad$ Methyl Methyl

${ }^{1} \mathrm{H}$ NMR $\left(250 \mathrm{MHz}, \mathrm{CDCl}_{3}\right) \delta(\mathrm{ppm}): 2.04,2.12$, $2.31\left(\mathrm{~s}, 9 \mathrm{H}, 3 \mathrm{CH}_{3}\right), 5.47\left(\mathrm{~s}, 2 \mathrm{H}, \mathrm{CH}_{2}\right), 7.11(\mathrm{~s}, 4 \mathrm{H}$, $\left.\mathrm{CH}_{\text {arom }}\right) .{ }^{13} \mathrm{CNMR}\left(62.5 \mathrm{MHz}, \mathrm{CDCl}_{3}\right) \delta$ (ppm): 11.58 , 12.87, $21.00\left(3 \mathrm{CH}_{3}\right), 50.53\left(\mathrm{CH}_{2}\right), 95.28$ (C of alkene), 126.66, 129.45 (4CH), 132.04, 137.00 (2C), 138.46 (C of alkene), $171.92(\mathrm{C}=\mathrm{S})$. Analysis of $\mathrm{C}_{13} \mathrm{H}_{15} \mathrm{NS}_{2}$ (249.39). (\% calculation/found): $\mathrm{C}: 62.61 / 62.55, \mathrm{H}$ : 6.06/6.00, N: 5.62/5.68.

\section{Results and discussions}

We examined the reaction of primary amines, carbon disulphide with $\alpha$-haloketone in the presence of potassium iodide in dry $\mathrm{CH}_{3} \mathrm{OH}$ at room temperature $\left(25^{\circ} \mathrm{C}\right)$ and we obtained the corresponding 3,4,5-trialkyl-1,3-thiazole-2(3H)-thione derivatives $\mathbf{4}$ in excellent yields. The reaction proceeds smoothly and cleanly under mild and neutral conditions, also no side products were observed. The compounds 4 were stable when stored at room temperature for several months.

The structures of products were deduced from their ${ }^{1} \mathrm{H}$ NMR, ${ }^{13} \mathrm{C}$ NMR, IR, and mass spectra. The mass spectra of these compounds displayed molecular ion peaks at the appropriate $m / z$ values. The ${ }^{1} \mathrm{HNMR}$ spectrum of $4 \mathbf{a}$ consisted of a singlet for $\mathrm{CH}_{2}$ at

Table 2. Synthesis of 3,4,5-trialkyl-1,3-thiazole-2(3H)thione $\mathbf{4 a}$ under various amounts of catalyst.

\begin{tabular}{lcccc}
\hline Entry & Catalyst (g) & Temp. $\left({ }^{\circ} \mathrm{C}\right)$ & Time (h) & Yield (\%) \\
\hline 1 & KI $(0.05)$ & r.t. & 7 & 50 \\
2 & KI $(0.07)$ & r.t. & 7 & 60 \\
3 & KI $(0.10)$ & r.t. & 7 & 85 \\
4 & KI $(0.15)$ & r.t. & 5 & 85 \\
5 & KI $(0.20)$ & r.t. & 5 & 85 \\
\hline
\end{tabular}

$\delta=5.34 \mathrm{ppm}$, two singlet for $2 \mathrm{CH}$ of furan at $\delta=6.12$ and $6.24 \mathrm{ppm}$, and a multiplet at $\delta=7.00-7.47 \mathrm{ppm}$ for eleven aromatic protons and furan proton. The ${ }^{1} \mathrm{H}$ decoupled ${ }^{13} \mathrm{C}$ NMR spectrum of $\mathbf{4 a}$ showed 16 distinct resonances, partial assignment of these resonances is given in section 2 . The ${ }^{1} \mathrm{H}$ and ${ }^{13} \mathrm{C}$ NMR spectra of compounds $\mathbf{4 b} \mathbf{b}-\mathbf{i}$ were similar to those of $\mathbf{4 a}$, except for the aromatic moiety, which exhibited characteristic signals with appropriate chemical shifts.

A possible mechanism for the present reaction is shown in scheme 2, which envisages a tandem sequence. On the basis of the established chemistry of trivalent nitrogen nucleophiles, the successful nucleophilic attack by amines on a carbon atom is facilitated when the latter is conjugated with a carbonyl group, or when it is a part of an otherwise activated unsaturated bond. First, the nucleophilic addition of the primary amine $\mathbf{3}$ to carbon disulphide $\mathbf{2}$ generates the nucleophilic carbamodithioic acid 5. ${ }^{26}$ The next step involves nucleophilic attack of carbamodithioic acid $\mathbf{5}$ at the carbon of haloketone 1, leading to intermediate 6, and then ring closure by intramolecular attack of nitrogen at the carbonyl carbon to afford the 3,4,5trialkyl-1,3-thiazole-2(3H)-thione derivatives 4.

Potassium iodide was found to catalyse the synthesis of 3,4,5-trialkyl-1,3-thiazole-2(3H)-thione

Table 3. Synthesis of 3,4,5-trialkyl-1,3-thiazole-2(3H)thione $\mathbf{4 a}$ in various solvents.

\begin{tabular}{lcccc}
\hline Entry & Solvent & Temp. $\left({ }^{\circ} \mathrm{C}\right)$ & Time (h) & Yield (\%) \\
\hline 1 & $\mathrm{H}_{2} \mathrm{O}$ & r.t. & 7 & - \\
2 & $\mathrm{C}_{2} \mathrm{H}_{5} \mathrm{OH}$ & r.t. & 7 & 50 \\
3 & $\mathrm{CH}_{3} \mathrm{OH}$ & r.t. & 5 & 85 \\
4 & $\mathrm{CH}_{2} \mathrm{Cl}_{2}$ & r.t. & 7 & 20 \\
5 & $\mathrm{CHCl}_{3}$ & r.t. & 7 & 20 \\
\hline
\end{tabular}


derivatives $\mathbf{4}$ from $\alpha$-haloketone $\mathbf{1}$, the primary amines $\mathbf{2}$, and carbondisulphide $\mathbf{3}$, in methanol (table 1). The use of just $0.1 \mathrm{~g}$ of potassium iodide (per mmol of reactants) is sufficient to push the reaction forward. Higher and lower amounts of potassium iodide (KI) did not considerably improve the yields (table 2). We also used other solvents in the presence of potassium iodide, but in all cases, increasing reaction times and decreasing yields of $\mathbf{4}$ were observed (table 3 ).

\section{Conclusion}

In this research work, a simple, mild and efficient method has been reported for preparation of 3,4,5-trialkyl-1,3-thiazole-2(3H)-thione derivatives. The products obtained in excellent yields under mild reaction and with simple work-up conditions. The products have been confirmed by physical and spectroscopic data such as; IR, ${ }^{1} \mathrm{HNMR},{ }^{13} \mathrm{CNMR}$, MS spectroscopy, and CHN elemental analyses.

\section{Supplementary information}

The electronic supporting information can be seen in www.ias.ac.in/chemsci.

\section{Acknowledgements}

Authors are thankful to the University of Kashan for supporting this work (by Grant NO: 159196/VII).

\section{References}

1. Zhu J and Bienaymé H (eds) 2005 Multicomponent reactions, Wiley- $\mathrm{VCH}$, Weinheim.

2. Ugi I, Werner B and Dömling A 2003 Molecules 853

3. Domling A 2006 Chem. Rev. 10617

4. Dömling A and Ugi I 2000 Angew. Chem. Int. Ed. 39 3168

5. Dömling A, Herdtweck E and Heck S 2006 Tetrahedron Lett. 471745

6. Ramazani A, Ahmadi Y and Tarasi R 2011 Heteroat. Chem. 2279

7. Ramazani A, Zeinali Nasrabadi F, Mashhadi Malekzadeh A and Ahmadi Y 2011 Monatsh Chem. 142625

8. Ramazani A, Farshadi A, Mahyari A, Ňlepokura K, Lis T and Rouhani M $2011 \mathrm{~J}$. Chem. Crystallogr. 41 1376

9. Ramazani A, Karimi Z, Souldozi A and Ahmadi Y 2012 Turk. J. Chem. 3681

10. Pessoa-Mahana H, Johann K.C, Nadia R.H, RecabarrenGajardo G, Claudio S. B, Araya-Matsurana R and Pessoa-Mahana C.D 2008 Heterocycles 751913

11. Blagg J, Mowbray C, Pryde D.C, Salmon G, Schmid E, Fairman D and Beaumont K 2008 Bioorg. Med. Chem. Lett. 185601
12. Simoni D, Romagnoli R, Baruchello R, Rondanin R, Grisolia G, Eleopra M, Rizzi M, Tolomeo M, Giannini G, Alloatti D, Castorina M, Marcellini M and Pisano C 2008 J. Med. Chem. 516211

13. Radwan M A A, Shehab M A and El-Shenawy S M 2009 Monatsh. Chem. 140445

14. Fakar I M I, Radwan M A A, El-Batran S, Abd El-Salam O M E and El-Shenawy S M 2009 Eur. J. Med. Chem. 441718

15. Abreu R M V, Ferreira I C F R and Queiroz M J R P 2009 Eur. J. Med. Chem. 441952

16. Aloup J C, Bouchaudon J, Farge D, James C, Deregnaucourt J and Hardy-Houis M 1987 J. Med. Chem. 3024

17. Gronowitz S 1985 Thiophenes and its derivatives; S Gronowitz Ed, New York: John Wiley and Sons, Part 142

18. Luker T J, Beaton H G, Whiting M, Kete A and Cheshire D. R 2000 Tetrahedron Lett. 417731

19. Roncali J 1997 Chem. Rev. 97173

20. Schopf G, Kossemehl G 1997 Adv. Polym. Sci. 1291

21. Dimitrakopoulos C D and Malenfant P R L $2002 A d v$. Mater. 1499

22. Morrison J J, Murray M M, Li X C, Holmes A B, Morratti S C, Friend R H and Sirringhaus H 1999 Synth. Met. 102987

23. Mashraqui S H, Hariharasubrahmanian $\mathrm{H}$ and Kumar S 1999 Synthesis 2030

24. Prim D, Joseph D and Kirsch G Liebigs 1996 Ann. Chem. 239

25. Augustine M, Rudorf W D and Schimdt U 1976 Tetrahedron 323055

26. Khalilzadeh M A, Hossaini Z, Baradarani M M and Hasannia A 2010 Tetrahedron 668464

27. Ramazani A, Ahmadi Y, Rouhani M., Shajari N and Souldozi A 2010 Heteroatom Chem. 21368

28. Ramazani A, Shajari, N, Mahyari A and Ahmadi Y 2011 Mol. Divers. 15521

29. Souldozi A and Ramazani A 2009 Phosphorus Sulfur Silicon 1843191

30. Ramazani A and Souldozi A 2009 Phosphorus Sulfur Silicon 1842344

31. Ramazani A, Zeinali Nasrabadi F, Karimi Z and Rouhani M 2011 Bull. Korean Chem. Soc. 322700

32. Ramazani A, Zeinali Nasrabadi F and Ahmadi Y 2011 Helv. Chim. Acta. 941024

33. Souldozi A, Ramazani A, Bouslimani N and Welter R 2007 Tetrahedron Lett. 482617

34. Souldozi A and Ramazani A 2007 Tetrahedron Lett. 48 1549

35. Ramazani A, Mahyari A T, Rouhani M and Rezaei A 2009 Tetrahedron Lett. 505625

36. Zeinali Nasrabadi F, Ramazani A and Ahmadi Y 2011 Mol. Divers. 15791

37. Ramazani A, Ahmadi Y and Zeinali Nasrabadi F 2011 Z Naturforsch. 66b 184

38. Ramazani A and Souldozi A 2009 Phosphorus Sulfur Silicon Relat. Elem. 1843191

39. Ramazani A and Rezaei A 2010 Org. Lett. 122852

40. Safaei-Ghomi J, Salimia F, Ramazani A, Zeinali Nasrabadi F and Ahmadi Y 2012 J. Sulfur Chem. 3387 